\title{
Importancia del servicio social para la formación de los profesionales de la salud. Contexto histórico
}

\author{
Importance of social service for the training of health professionals. Historic context
}

\author{
Aldo Pelcastre-Neri ${ }^{a}$, Brenda E. Barraza-Sánchez ${ }^{b}$, Mario Diego-Martínez ${ }^{c}$, Josefina \\ Reynoso-Vázquez ${ }^{d}$, Antonia Y. Iglesias-Hermenegildo ${ }^{e}$, Jesús C. Ruvalcaba-Ledezma $^{e^{*}}$
}

\begin{abstract}
:
University social service is a series of activities where you conclude your professional training and put into practice the academic knowledge learned throughout. The focus is to help and answer societies demands to health issues. For its part, the Regulatory Law of Article 5. Constitutional, Relating to the Exercise of Professions in the Federal District, the Social Service, as temporary work and the compensation that is executed and provided to professionals and students in the interest of society and the State.

That is why it is considered essential to conduct a brief review of social service in Mexico and the Autonomous University of the State of Hidalgo, and to take into account the importance of the development of professionals in the field of health.

Keywords:

Social Service, health, professional training

\section{Resumen:}

Hablar de servicio social universitario es hacer referencia a una serie de actividades donde el alumno que se encuentra por concluir su formación profesional pone en práctica los conocimientos académicos adquiridos a lo largo de esta, a fin de brindar atención a las demandas en salud de la sociedad, pudiendo provenir de instituciones públicas o privadas, sobre todo en lugares donde la atención es nula. Por su parte la Ley Reglamentaria del Artículo 5o. Constitucional, Relativo al Ejercicio de las Profesiones en el Distrito Federal define al servicio social como el trabajo de carácter temporal y mediante retribución que ejecuten y presten los profesionistas y estudiantes en interés de la sociedad y el Estado.

Es por ello que se considera fundamental realizar una breve revisión histórica del servicio social en México y de la Universidad Autónoma del Estado de Hidalgo, ya que esto permitirá dar cuenta de la importancia de este para el desarrollo de los profesionales del campo de la salud.
\end{abstract}

Palabras Clave:

Servicio Social, salud, formación profesional

\footnotetext{
${ }^{a}$ Universidad Autónoma del Estado de Hidalgo, Instituto de Ciencias de la Salud, Maestría en Salud Pública: life_9422@hotmail.com

b Universidad Autónoma del Estado de Hidalgo, Instituto de Ciencias de la Salud, Maestría en Salud Pública: brenbarrz@hotmail.com

${ }^{\mathrm{c}}$ Universidad Autónoma del Estado de Hidalgo, Instituto de Ciencias de la Salud, Maestría en Salud Pública: psic.mdm@gmail.com

d Área Académica de Farmacia y Maestría en Salud Pública del Instituto de Ciencias de la Salud, Universidad Autónoma del Estado de Hidalgo: jreynoso@uaeh.edu.mx

Área Académica de Psicología, Instituto de Ciencias de la Salud, Universidad Autónoma del Estado de Hidalgo: an.iglesias13@gmail.com

${ }^{\mathrm{f}}$ Autor de Correspondencia, Área Académica de Medicina y Maestría en Salud Pública, Instituto de Ciencias de la Salud, Universidad Autónoma del Estado de Hidalgo, ORCID: 0000-0001-1234-1234, Email: dcspjcarlos@ gmail.com
} 


\section{INTRODUCCIÓN}

Hablar de servicio social universitario es hacer referencia a una serie de actividades donde el alumno que se encuentra por concluir su formación profesional pone en práctica los conocimientos académicos adquiridos a lo largo de esta, a fin de brindar atención a las demandas en salud de la sociedad, pudiendo provenir de instituciones públicas o privadas, sobre todo en lugares donde la atención es nula. ${ }^{(1)}$ Por su parte la Ley Reglamentaria del Artículo 5o. Constitucional, Relativo al Ejercicio de las Profesiones en el Distrito Federal define al servicio social como el trabajo de carácter temporal y mediante retribución que ejecuten y presten los profesionistas y estudiantes en interés de la sociedad y el Estado. ${ }^{(2)}$

Es por ello que se considera fundamental realizar una breve revisión histórica para del servicio social en México y de la Universidad Autónoma del Estado de Hidalgo, ya que esto permitirá dar cuenta de la importancia de éste para el desarrollo de los profesionales del campo de la salud. ${ }^{(3)}$

\section{Importancia del Servicio Social}

En el área de la Salud, el Servicio Social toma relevancia en relación a las oportunidades que el alumno en formación puede obtener. Como ya se ha mencionado, el servicio social pretende que el futuro profesional de la salud ponga en práctica y consolide toda una serie de conocimientos, habilidades, capacidades y destrezas durante la atención de salud de calidad brindada a la sociedad en alguna institución u organización pública o privada. Asimismo, la prestación del Servicio Social brinda oportunidad de acercar de manera directa al pasante a obtener un empleo, vincularlo con diversos sectores de la salud $y$ relacionarlo para saber trabajar de manera interdisciplinaria en el ámbito profesional. Sin embargo, el contar con todas las oportunidades de desempeño profesional en el Servicio Social, la vinculación de la universidad con instituciones del sector salud y toda una serie de acciones de la institución educativa que permitieron consolidar oportunamente esta práctica, se debe a toda una serie de hechos históricos que tuvieron gran auge a nivel nacional y local, donde paso a paso se logró que los alumnos pudieran tener una experiencia basta en brindar un servicio de salud apegado a cada disciplina. ${ }^{(4)}$ Es por esto que se debe conocer la historia del Servicio Social para entenderlo, según la UAEH, como un "elemento de trascendencia estratégica para estrechar los vínculos con los ciudadanos y sectores, ayudando a transformar de manera dinámica y propositiva el desarrollo comunitario".(5)

\section{Servicio Social en México}

El 22 de septiembre de 1910, en la conmemoración del primer centenario del inicio de la Guerra de Independencia, la Universidad Nacional, ahora Universidad Nacional Autónoma de México, reabre sus puertas a la comunidad estudiantil. A partir de este acontecimiento la Ley Constitutiva de la Universidad Nacional de México elabora dos artículos importantes en materia de extensión universitaria, el Artículo $1^{\circ}$ donde menciona que el objeto primordial de la Universidad es "realizar la obra educativa nacional" y en el Artículo $8^{\circ}$ estableció que el Consejo Universitario debe organizar lo que se denomina extensión universitaria. Así, los fines estipulados en aquella ley al restablecerla fueron: la educación y la extensión universitaria. Durante el rectorado de José Vasconcelos en la UNAM, en el periodo de 1920-1921, uno de los departamentos universitarios que tuvieron mayor éxito fue precisamente el de Extensión Universitaria. El entonces rector incorporó a los estudiantes a realizar tareas relacionadas con extensión universitaria y a campañas de alfabetización. Durante el año de 1926, cuando fue rector Alfonso Pruneda (periodo 1924-1928) hubo una crisis financiera dentro de la universidad y fue en relación al presupuesto de aquel año donde lo destinado a la Universidad nunca apareció. En consecuencia, profesores salieron a protestar y debido a su gran responsabilidad con los alumnos, decidieron trabajar gratuitamente por el tiempo que fuera necesario sumando que iniciaron campañas de instrucción cívica, 
conferencias de cultura general, bufetes jurídicos con los estudiantes de Derecho y dispensarios médicos con los de Medicina, todos ellos coordinados por el Departamento de Extensión Universitaria. Los planteamientos iniciales de la idea de un servicio destinado a la sociedad en México iniciaron en 1929 y fueron ideas de estudiantes pertenecientes a la hoy Universidad Nacional Autónoma de México que luchaban por la autonomía universitaria y por maestros como Antonio Caso y Enrique Gómez Morín. Por otra parte, Justo Sierra fue uno de los primeros en plantear justificadamente que la educación que imparte cada universidad no debe limitarse a aplicar soluciones a las necesidades de la sociedad, sino que debe de buscar, mediante la enseñanza y la investigación científica, soluciones con medios que estén al alcance para contribuir en todo momento al desarrollo nacional. Cuando se inició formalmente el servicio social en la Universidad Nacional era presidente de la república el general Lázaro Cárdenas (1934-1940). ${ }^{(6)(7)(8)}$

En 1933, cuando era Rector Enrique Gómez Morín además de ser defensor de la libertad de cátedra, luchó a diario por librar a la Universidad de la situación económica difícil por la que estaba pasando. El seis de marzo de 1934 el periódico "El Universal" sacaba una noticia digna de dar a conocer en la cual mencionaba que la Rectoría de la Universidad enviaría al Congreso de Profesionistas, reunido en la $\mathrm{Cd}$. de México, la iniciativa de establecer el servicio social. Las ideas sociales de la Revolución Mexicana, con respecto al servicio social, se vieron reflejadas en la Constitución Mexicana, ya que en los artículos cuarto y quinto, se establecía que los profesionistas deben ser útiles a la sociedad. ${ }^{(8)(9)}$ Para el año de 1935, la cuestión se hizo particularmente crítica, ya que en ese año se reformó el artículo 3o, según el cual la educación que impartiera el Estado tendría que ser completamente socialista, lo que provocó de inmediato la reacción de los universitarios como Antonio Caso que pensaban que la Universidad no podría restringirse a una sola doctrina, sin traicionar sus principios. ${ }^{(6)}$ El Dr. Gustavo Baz Prada, en respuesta a las personas que pedían el cierre de la Universidad, presentó el 2 de diciembre de 1935, al rector Luis Chico Goerne el proyecto que tenía por objetivo que los estudiantes de la escuela Nacional de Medicina pudieran prestar servicio social. Después de toda la gestión y revisión, en 1936 se implementó el servicio médico social al celebrarse el primer convenio entre una dependencia del sector público, el Departamento de Salubridad Pública y la Escuela Nacional de Medicina de la UNAM. En 1936 el doctor Gustavo Baz Prada, al saber las necesidades de los campesino por haber tenido un estrecho contacto con ellos, organizó la primer brigada multidisciplinaria, que reunió a estudiantes de las escuelas de Medicina, Biología, Odontología, Veterinaria, Ingeniería, Arquitectura, Artes Plásticas, Música, Derecho y Química, quienes fueron a atender durante un periodo de cinco meses a obreros, campesinos y a la población en general de Atlixco, Puebla. En el mismo año, el doctor Gustavo Baz, aun siendo director de la Escuela de Medicina, instauró el servicio social con carácter obligatorio para los pasantes de esa carrera, y para el año de 1938, siendo rector de la UNAM, lo hizo obligatorio para todos los pasantes de ésta universidad. ${ }^{(8)}$

En agosto de 1936 los primeros 248 pasantes de la carrera de médico cirujano salieron a prestar servicio social. Iban con un equipo modesto en un maletín médico, con un lote de diversos productos biológicos y con una pequeña remuneración de noventa pesos mensuales. Fueron a establecerse por cuatro o cinco meses a diversos rincones del país, previamente señalados y que les fueron repartidos de acuerdo con sus deseos. Por otra parte, en 1944 se inicia formalmente el servicio social en el Instituto Politécnico Nacional, incorporándose por primera vez a esta gran labor la carrera de enfermería y en cumplimiento a la Ley Reglamentaria del artículo 5o. Constitucional publicada en 1945, se fueron agregando diversas carreras de la salud como odontología y posteriormente las demás. A partir de 1945 la Ley Reglamentaria del Artículo 5을 establece la diferenciación entre el servicio social de estudiantes y el de profesionales, y señala que 
para los primeros la responsabilidad corresponde a la Institución Educativa. ${ }^{(10)}$

En 1978, por acuerdo presidencial, se creó la Comisión Coordinadora del Servicio Social de Estudiantes de las Instituciones de Educación Superior (Cossies), la cual surgió ante la necesidad de establecer mecanismos de coordinación interinstitucional entre las acciones del gobierno en materia de servicio social. Por esta razón, la Cossies desarrolló un Plan General de Servicio Social porque una de sus facultades era la de coordinar la planeación y la programación presupuestal de las acciones nacionales de servicio social. ${ }^{(7)}$

En 1979 como producto de la Primera Reunión Nacional de Intercambio sobre Servicio Social, realizado en la Universidad de Guanajuato, se propone la creación de la Comisión Interuniversitaria de Servicio Social la cual tendría como objetivo la elaboración del marco metodológico para el desarrollo de esta actividad a través de la Comisión Interuniversitaria de servicio social (CISS). En el "Reglamento para la Prestación del Servicio Social de los Estudiantes de las Instituciones de Educación Superior de la República Mexicana" publicado en el Diario Oficial de la Federación en 1981, se refuerza el planteamiento educativo y se señala que debe formar parte del plan de estudios correspondiente, además, establece las bases y lineamientos para la realización de esta actividad a través del Sistema Nacional de Servicio Social con la coordinación de COSSIES. Con base en este Reglamento, se publicaron en el mismo diario en 1982, las "Bases para la Instrumentación del Servicio Social en el Sector Salud" pero con un enfoque principalmente asistencial. En marzo de 1984 el Comité de Enseñanza de Pregrado y Servicio Social tenía entre sus programas de trabajo, la elaboración de una propuesta de reglamento de servicio social para las carreras de la salud en el marco de la Ley General de Salud publicada en el diario oficial de la federación el 7 de febrero de 1984 y en la que se sustituyó a la Secretaría de Salubridad y Asistencia por la Secretaría de Salud. Al desaparecer COSSIES surge en su lugar en 1985, al interior de la Secretaría de Programación y Presupuesto, la Dirección de Apoyo al
Servicio Social de los Estudiantes y de Análisis del Empleo (DASSEAE), misma que en 1989 se transformó en Dirección de Apoyo al Servicio Social (DASS) instancia que pasa a formar parte de la estructura de la Secretaría de Desarrollo Social (SEDESOL) durante el sexenio de 1989 al $1994 .^{(10)}$

\section{Antecedentes de Servicio Social de la UAEH}

La Dirección de Servicio Social de la Universidad Autónoma del Estado de Hidalgo se crea el 6 de agosto de 1971 por acuerdo del Honorable Consejo Universitario. Su función principal es ofrecer la posibilidad a los alumnos que están por concluir su formación académica participar en actividades teóricopráctica de manera temporal, volviéndose así de carácter obligatorio. En mayo del año 2006 con la reestructuración organizacional de la $U A E H$ se constituye la Dirección de Servicio Social y Prácticas Profesionales. ${ }^{(11)}$

La Dirección de Servicio Social y Prácticas Profesionales tiene como propósito vincular a los alumnos con organizaciones, instituciones y dependencias de los sectores público, privado y social a nivel nacional e internacional, en los cuales puedan poner en práctica los conocimientos adquiridos durante su formación académica y desarrollen así, habilidades y competencias genéricas y específicas que contribuyan para su inserción al campo laboral. Así pues, el servicio social se ha convertido en una herramienta clave en la articulación del estudiante con la sociedad a través de programas comunitarios, en los cuales se realizan acciones de asistencia social enfocadas a la salud, educación y cuidado del medio ambiente. Los programas institucionales de servicio social administrados en esta dirección son: Universitarios en Acción, Unidades de Desarrollo Comunitario, Universitarios por la Alfabetización, Cuenta Conmigo y Servicio Social Voluntario, los cuales enfocan su atención a la población de las zonas de mayor rezago de pobreza en los 84 municipios del estado. ${ }^{(12)}$ 


\section{Conclusión}

El contexto histórico del servicio social tiene que ser de mayor importancia para el conocimiento de todos los alumnos que van a realizar servicio social en carreras de la salud. Esto es importante teniendo en cuenta que esta práctica profesional tiene una relevancia tanto para el futuro profesional de la salud como para la sociedad a la que va dirigida la atención en salud; por un lado el profesional de la salud en formación tiene un campo en donde desarrolla habilidades y obtiene conocimientos propios de la práctica clínica y por otro lado aprende a brindar una atención de calidad a los usuarios en los diversos servicios.

\section{REFERENCIAS}

[1] Autónoma U. Departamento De Servicio Social Y Becas. [citado el 23 de noviembre de 2017];1. Disponible en: https://www.uaeh.edu.mx/adminyserv/gesuniv/div_vin/dir_sspr act/ss/index.html

[2] González-Ramírez P. Nivel de cumplimiento de las expectativas de formación profesional de los programas de servicio social para los pasantes de la licenciatura en Enfermería y Obstetricia. México D.F.; 2012. 117 p.

[3] Cámara de Diputados. LEY REGLAMENTARIA DEL ARTíCULO 5o. CONSTITUCIONAL, RELATIVO AL EJERCICIO DE LAS PROFESIONES EN EL DISTRITO FEDERAL. Diario Oficial de la Federación [Internet]. 1993 [citado el 17 de enero de 2018];1:18. Disponible en: http://www.oas.org/juridico/spanish/mesicic3_mex_ley_regl_ar t_5.pdf

[4] México UNA de. ABC del Servicio Social [Internet]. 2016 [citado el 22 de febrero de 2019]. p. 1. Disponible en: http://www.siients.unam.mx/serviciosocial/inicio.php?clave=A BCSS

[5] UAEH. Servicio Social Universitario [Internet]. [citado el 22 de febrero de 2019]. Disponible en: https://www.uaeh.edu.mx/adminyserv/gesuniv/div_vin/dir_sspr act/ss/index.html

[6] Ramírez CL. El entorno histórico en el que se dio el Servicio Social. Gaceta Médica de México [Internet]. 2012;1(Servicio Social):281-3.

Disponible
http://www.anmm.org.mx/GMM/2012/n3/GMM_148_2012_3 _281-283.pdf

[7] Bárcena MR, Elisa M, Barragán C, García CN, Rossi L. El servicio social. Dirección General de Orientación y Servicios Educativos, UNAM. 2012;1:237-44.

[8] Rodríguez, Yolanda et al. Caminos y Rumbos del Servicio Social en México [Internet]. 1a ed. Universidad Iberoamericana, Universidad Intercontinental, Universidad Pedagógica Nacional, editores. México ; 1997 [citado el 19 de febrero de 2019]. 1-198 p. Disponible en: http://campus.ajusco.upn.mx:8080/upn/bitstream/handle/11195 /458/Caminos y rumbos del servicio social en México.pdf?sequence=1

[9] Gil C, Bautista O, Pancardo P, Hernández J, Cruces E, Ronzón J, et al. 28 Congreso de Servicio Social, Memoria Electrónica. Fondo Edit. Bautista, Campos et al., editor. Vol. 1, Universidad Juárez Autónoma de Tabasco. Tabasco, México: Colección; 2011. 1-891 p.

[10] López J. Servicio Social de Medicina en México. Factibilidad del cumplimiento académico en el área rural Derechos. Revista de la Facultad de Medicina. 2004;47:181-6.

[11] Dirección de Servicio Social PP y VL. Antecedentes [Internet]. Página Oficial UAEH. 2016 [citado el 12 de junio de 2018]. p. 1. Disponible en: https://www.uaeh.edu.mx/adminyserv/gesuniv/div_vin/dir_sspr act/antecedentes.html

[12] Dirección de Servicio Social PP y VL. Propósito [Internet]. Página Oficial UAEH. [citado el 12 de junio de 2018]. p. 1. Disponible en: https://www.uaeh.edu.mx/adminyserv/gesuniv/div_vin/dir_sspr act/bienvenida.html 